\title{
Stability of handwriting performance following injury-induced hand- dominance transfer in adults: A pilot study
}

\author{
Kathleen E. Yancosek, PhD, OTR/L, CHT; ${ }^{1 *}$ David R. Mullineaux, PhD $^{\mathbf{2}}$ \\ ${ }^{1}$ Department of Rehabilitation Sciences, College of Health Sciences, University of Kentucky, Lexington, KY; \\ ${ }^{2}$ Department of Kinesiology and Health Promotion, College of Education, University of Kentucky, Lexington, KY; \\ and Sport, Coaching, and Exercise Science, University of Lincoln, Brayford Pool, Lincoln, UK
}

\begin{abstract}
The aim of this study was to quantify stability of nondominant handwriting kinematics and legibility in participants with functional loss of the previously dominant hand. Twelve adult volunteers provided two handwriting samples 6 weeks apart. Handwriting tasks (Compose a Sentence, Copy Alphabet, Copy Date, Copy Sentence, and Draw Circles) were performed in cursive writing on standard white, lined paper taped to a digitizer to record kinematic and kinetic variables of velocity, displacement, force, and on-paper time. Results showed minimal performance variability within subjects and marked variability between subjects, as well as variability between tasks for all participants. Stylistic stability of the handwriting samples was assessed by two independent evaluators. These evaluators matched all handwriting samples at test to retest times with $89 \%-100 \%$ accuracy, suggesting value in the "whole" handwriting sample and emphasizing the idiosyncratic nature of handwriting. Results suggest that handwriting skill stability in the previously nondominant hand varies across subjects and task demands.
\end{abstract}

Key words: activities of daily living, amputation, handwriting, hand dominance, injury-induced hand-dominance transfer, Jebsen-Taylor Test of Hand Function, kinematics, performance, reliability, stability.

\section{INTRODUCTION}

Most activities of daily living (ADL) are accomplished bimanually with the dominant hand as main executor and the nondominant hand as supporter [1]. When normal bilateral hand function is disrupted [2], patients must complete two-handed tasks with one hand. A functional state of "single-handedness" may be temporary, such as is common in recovery from tendon laceration and repair, fracture and fixation, or neuropraxia and splinting; however, when prognosis for functional return is poor, a permanent state of single-handedness ensues. This onehanded situation is more difficult with dominant-hand impairment because complex fine-motor coordination and skills must be transferred to the nondominant hand [3].

A forced shift of dominance is termed injury-induced hand-dominance transfer (I-IHDT). It conceptually defines the imposed transfer of lateralized skill proficiency to the previously nondominant hand. Besides amputation of a dominant upper limb, other diagnoses potentially result in single-handedness and I-IHDT, such as brachial plexus avulsion, chronic unilateral lymphodema, hemiparesis following stroke, focal hand dystonia, limb salvage following mutilating hand injury (crush,

Abbreviations: $\mathrm{ADL}=$ activity of daily living, $\mathrm{CI}=$ confidence interval, ICC $=$ intraclass coefficient correlation, I-IHDT $=$ injury-induced hand-dominance transfer, JTHF $=$ Jebsen-Taylor Test of Hand Function, SD = standard deviation.

*Address all correspondence to MAJ Kathleen E. Yancosek, PhD, OTR/L, CHT; U.S. Army Research Institute of Environmental Medicine, 15 Kansas Street, Building 42, Natick, MA 01760; 508-233-5454.

Email: kathleen.yancosek@us.army.mil

DOI:10.1682/JRRD.2010.04.0074 
avulsion, burns), and complex regional pain syndrome following minor trauma or surgery [4].

Hand dominance is closely associated with, and often defined by, the functional neuromotor task of handwriting [5]. Handwriting, as a form of functional dexterity, captures the hand's interface with a commonly encountered tool. Handwriting also captures the hand's intricate link to the brain for planning and executing purposeful movements, in this case, written expression [6-7]. Because handwriting is purported to be the highest form of unilateral hand dexterity skill attained by the general population [8], it is an important component of I-IHDT.

Handwriting is a distinct neuromotor skill of interest to occupational therapy practitioners. The Handwriting Assessment Battery for adults evaluates pen control and manipulation, writing speed, and writing legibility [9]. Writing is one of seven functional tasks on the JebsenTaylor Test of Hand Function (JTHF) [10]. Handwriting is included in many self-report questionnaires on hand function; for example, handwriting is a specific item listed on the Disabilities of the Arm, Shoulder, and Hand questionnaire [11]; signing one's name is included in the physical domain portion of the Burn Specific Health Scale [12]; and the Upper Limb Functional Index includes the item "I have difficulty writing or using a key board and/or mouse” [13].

Beyond self-rated scales, there is a need for better quantification of fine-motor control needed for handwriting [14]. The field of graphonomics provides technology for quantifying handwriting (graphomotor) performance. This type of digital analysis was used to capture disturbed motor control in patients with chronic undiagnosed wrist pain [15]. Leveraging digital technologies and using graphonomics as the portal to evaluate dexterity performance has clinical implications for the evaluation of the process and outcome of I-IHDT.

\section{Literature Review}

Many diagnoses may lead to I-IHDT; however, a limited body of literature exists. Chan and LaStayo, in their description of the management of mutilating hand injuries, recommend early instruction in ADL, specifically if a dominant hand is injured [16]. Research on neuroplasticity, motor learning, and intermanual transfer informs clinical practice; however, these studies are generally limited by their use of simple, nonfunctional motor tasks and/or recruitment of only nondisabled participants. One study evaluated 10 young, nondisabled adults who learned to write one character of a foreign alphabet with both hands [17]. The investigators concluded that occupational therapy practitioners should select tasks that are meaningful and previously known to the person to best facilitate the transfer. Another study on cross-dominance training required 21 nondisabled adults to repeatedly copy the same sentence daily for 28 consecutive days [18]. Results demonstrated that participants, 20 to 56 years old, gained proficiency in nondominant handwriting with no decrement from increasing age. The investigators did not test for generalization of handwriting skill by assessing performance on novel handwriting tasks.

A cohort-controlled neuroimaging study examined 16 adults who self-reported being "innately left-handed" but forced at the onset of school to convert to righthandedness. The study showed two cortical areas that correlated with handedness, and one area was more invariant than the other, regardless of sensorimotor training [19]. The researchers concluded that despite learning to write with the right hand, these 16 research subjects maintained a right-hemisphere dominance in the inferior parietal cortex and the rostrolateral premotor cortex. An additional neuroimaging study in humans found small, distinct writing centers in the brain, but they were specific and highly individualized for each of 14 subjects [20].

Taken together, these behavioral and imaging studies demonstrate training effects, perhaps despite central nervous system fixation of hemisphere dominance, thereby suggesting that neuromotor plasticity in relation to handwriting is more of a peripheral phenomenon.

\section{Purpose}

The primary aim of this investigation was to assess graphomotor performance consistency of adults who had lost hand function through amputation or permanent multitissue damage to the dominant upper limb more than 2 years ago. The assumption was made that by 2 years postinjury, participants would have achieved a general level of single-hand function and subsequent dominance transfer.

\section{METHODS}

Participants were primarily recruited via letters mailed through local hand-therapy and prosthetic centers. 
A secondary recruitment strategy was announcements about the study through a local amputee support group.

A one-group test-retest design was used in which participants provided two handwriting samples 6 weeks apart. No intervention was provided in this study. Six weeks was chosen as the time interval between assessments to accommodate future data comparison from planned clinical trials involving a 6-week handwritingskill transfer intervention.

Twelve adults volunteered and provided written informed consent. Three participants were excluded from analysis: an 84-year-old with notable tremor during writing tasks, a female with bilateral upper-limb amputations who wrote with a prosthesis, and a male who had undergone ray resection of the two most ulnar digits of his nondominant hand. Data from nine participants (3 males, 6 females; aged 27-70 years, mean: 53.6 years) were analyzed. No participant withdrew from the study. All participants lost function of the right, dominant hand with an average time since loss of function of 15 years (range: 3-46 years). Eight participants were amputees, and one participant had an attached but deformed and nonfunctional upper limb. See Figure 1 for select examples of participants.

Mechanism of injury was trauma for seven participants, multi-organ system failure for one participant, and localized blood clots with subsequent tissue necrosis and amputation in one participant. Six participants were retired, and three worked full-time. Eight participants reported daily engagement in handwriting tasks (average of 24 minutes a day). Participants who wore glasses for reading used them during the experiment. To increase study recruitment and enrollment, the investigators accommodated participants who did not drive by meeting them at a convenient location. Participants performed all graphomotor activities from a seated position. They were free to angle the writing apparatus according to preference; however, regardless of stylistic preference, they were asked to complete the handwriting activities in cursive, not manuscript, form. The decision to have participants write in cursive was made to accommodate future data comparison from planned clinical trials using the available handwriting intervention that instructs in cursive.

After three practice trials for familiarization, each participant completed the following six handwriting tasks: (1) Compose a Sentence, (2) Copy Alphabet, (3) Copy Date, (4) Copy Sentence 1, (5) Copy Sentence 2, and (6) Draw Circles. The Copy Alphabet and Draw Circles tasks were the same at test and retest sessions; however,

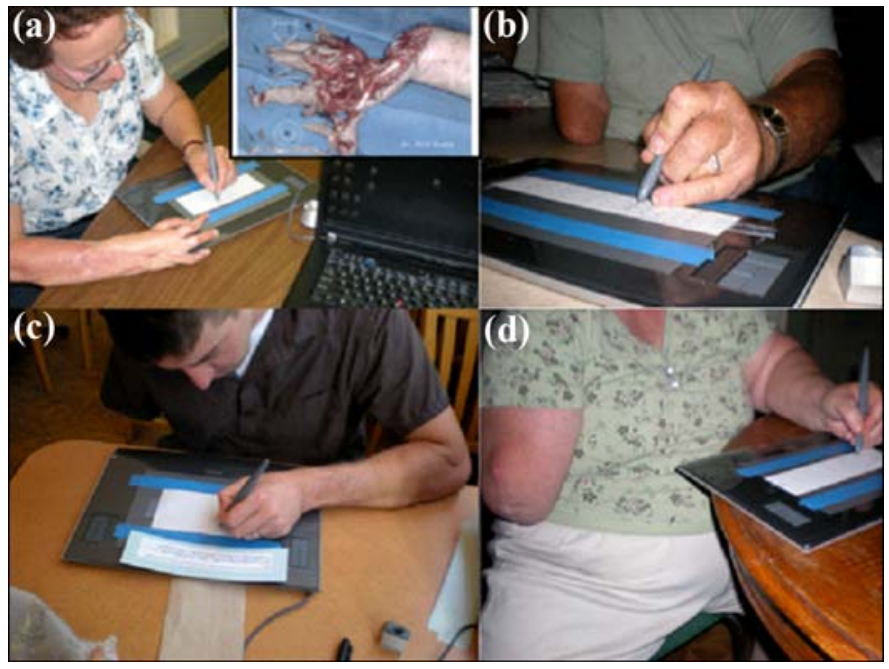

Figure 1.

Examples of participants: (a) female with mutilated hand injury, (b) male with high transradial amputation, (c) male with transhumeral amputation, and (d) female with elbow disarticulation.

Compose a Sentence, Copy Sentence 1, and Copy Sentence 2 were purposefully varied between sessions to diminish effects from memory and learning of experimental tasks. Each writing task was presented visually on a 2-inch card mounted on blue cardstock paper placed in front of the participant. The card contained the instructions (which were also read to the participant) and an example of the completed writing activity in cursive.

Graphomotor output during each of the six tasks was collected with use of a $3.5 \times 7.0$-inch piece of white lined paper taped to a digitizer tablet (Wacom Intuos 3, model PTZ-630, Wacom Company; Vancouver, Washington) controlled by a Lenovo ThinkPad notebook computer (Lenovo; Morrisville, North Carolina). MovAlyzR ${ }^{\circledR}$ software by NeuroScript ${ }^{\mathrm{TM}}$ (Tempe, Arizona) was used to set up, run the experiment, and capture the pen-tip kinematic data (left to right and top to bottom; paper position, i.e., $x$ and $y$ directions) and kinetic data (pen tip on paper force) at a sampling rate of $200 \mathrm{~Hz}$. The Intuos 3 Inking Pen (Wacom Company) was used as the wireless writing instrument. This apparatus offered a pen-on-paper feel with the benefits of direct digital recording of the pen-tip position and force. Customized code written with MATLAB ${ }^{\circledR}$ software (MathWorks; Natick, Massachusetts) was used to calculate further kinematic variables and calibrate the kinetic parameters of each handwriting activity. The following parameters were collected: force (grams), average 
displacement in the $x$ and $y$ directions (centimeters), average velocity of the pen tip in the $x$ and $y$ directions (centimeters per second), and on-paper time (seconds). These variables were selected because of their commonplace use in graphonomics research.

In addition to kinematic and kinetic variables, stylistic stability of handwriting samples served as another metric of performance consistency. After data were collected, handwriting samples were trimmed to remove participants' identification codes and mounted to card stock. The identification codes were rewritten on the back of the card stock. The principal investigator met separately with two objective evaluators who were uninvolved in the research study. One evaluator was a high-school administrator and one was a homemaker who previously worked as a behavioral health professional. Neither was experienced in handwriting assessment nor knowledgeable about the study objectives.

The investigator sequentially presented writing samples for all participants from six writing tasks by making two columns of the writing samples in random order. One column contained test samples for all participants, and the second column contained retest samples. The evaluators were instructed to visually inspect and correctly pair the handwriting samples thought to be written by the same participant (one from the test column and one from the retest column). After each evaluator made nine pairs, their results were calculated and recorded as the number of correct responses out of nine.

Kinematic and kinetic data in MATLAB were trimmed to 90 percent to cater for extreme pen movements (e.g., dotting an i). In SPSS (version 16, SPSS, Inc; Chicago, Illinois), data were then tested for normality (Shapiro-Wilks > 0.05) and outliers were removed. The test and retest data were evaluated for analysis by using the intraclass correlation coefficient (ICC).

To score and equate each participant's handwriting fluency to a grade-school level, the total task time for writing Copy Sentence 1 ("Don't question my mother, Zada K. Bigley, who is exceptionally virtuous, fashionable, and joyful.”) was converted to a written-letters-perminute score. This sentence was rated at an adult level (13.4 grade level) according to the Flesch-Kincaid scale, a widely used tool for assessing reading and writing complexity [21]. The number of letters in the sentence (77) was multiplied by 60 seconds and then divided by the number of seconds each participant took to complete the task. This score was then compared to the handwriting fluency numbers of a large sample $(n=900)$ provided by
Graham et al. of school-aged children from first to ninth grade [22].

The JTHF is a well-known hand-function assessment with seven subtests [10]. One subtest measures the time the adult takes to copy a sentence with 24 characters. The Copy Alphabet task in this pilot study required participants to copy (in cursive without spaces between letters) the 26 characters of the alphabet. Because this handwriting activity closely matched the writing subtest of JTHF, task completion time was examined for each participant and compared with normative data of the nondominant and dominant hands provided by original data from the JTHF [10].

\section{RESULTS}

The various kinematic and kinetic data showed different stability over the 6-week period. Table 1 shows mean and standard deviation (SD) values for all six writing tasks at test and retest sessions. Calculating the differences between test and retest measurements revealed relatively small group mean differences, which demonstrate a trend of within-subject performance stability; however, between-subject variability is indicated by the large SDs around the group means. Mean velocity (in the $x$ direction) was the most stable parameter and force the least stable between testing sessions across all six tasks. Draw Circles, Copy Date, and Copy Alphabet were the most consistently performed tasks across participants, whereas Compose a Sentence, Copy Sentence 1, and Copy Sentence 2 showed more variability across participants for all parameters. Velocity in the $x$ and $y$ directions was higher at retest for all tasks despite longer on-paper time for Copy Alphabet, Copy Sentence 1, and Copy Sentence 2. Force was consistently greater at the retest session for all tasks.

Table 2 shows reliability analysis of data by quantification methods using ICC for graphomotor performance from test to retest. The following kinematic parameters across the six tasks showed excellent correlation $(0.80$ 1.00): mean velocity in the $x$ direction for Copy Date, mean velocity in the $y$ direction for Draw Circles, and onpaper time for Copy Alphabet. The kinematic parameters with the highest correlation between test and retest sessions across all tasks were mean velocity in the $x$ direction and on-paper time; however, no single writing task had good to excellent correlation across all kinematic and kinetic parameters. 
Table 1.

Handwriting kinematics and kinetics at test and retest (mean \pm standard deviation) for six writing tasks completed with left hand by nine participants with permanent loss of function in previously dominant right hand. Gray shading denotes retest values.

\begin{tabular}{|c|c|c|c|c|c|c|}
\hline \multirow{2}{*}{ Task } & \multicolumn{2}{|c|}{ Mean Velocity $(\mathrm{cm} / \mathrm{s})$} & \multicolumn{2}{|c|}{ Displacement } & \multirow{2}{*}{$\begin{array}{l}\text { Force } \\
(g)\end{array}$} & \multirow{2}{*}{$\begin{array}{c}\text { On-Paper } \\
\text { Time (s) }\end{array}$} \\
\hline & $x$ & $y$ & $x$ & $y$ & & \\
\hline Compose Sentence & $0.83 \pm 0.50$ & $1.01 \pm 0.50$ & $6.18 \pm 0.80$ & $1.41 \pm 0.68$ & $68.14 \pm 41.46$ & $49.95 \pm 28.13$ \\
\hline Compose Sentence & $0.76 \pm 0.53$ & $0.97 \pm 0.49$ & $7.10 \pm 1.64$ & $1.27 \pm 0.39$ & $110.78 \pm 63.32$ & $45.96 \pm 21.97$ \\
\hline Copy Alphabet & $0.53 \pm 0.30$ & $0.66 \pm 0.32$ & $8.43 \pm 2.48$ & $0.44 \pm 0.11$ & $122.66 \pm 63.18$ & $47.56 \pm 42.33$ \\
\hline Copy Date & $0.81 \pm 0.45$ & $0.93 \pm 0.43$ & $4.71 \pm 2.29$ & $0.41 \pm 0.14$ & $74.28 \pm 40.49$ & $16.51 \pm 13.49$ \\
\hline Copy Sentence 1 & $0.64 \pm 0.38$ & $0.79 \pm 0.37$ & $7.43 \pm 1.51$ & $1.66 \pm 0.57$ & $103.65 \pm 59.55$ & $99.12 \pm 74.97$ \\
\hline Copy Sentence 2 & $0.70 \pm 0.37$ & $0.93 \pm 0.47$ & $7.22 \pm 1.35$ & $1.99 \pm 0.91$ & $68.03 \pm 29.37$ & $93.87 \pm 83.36$ \\
\hline Copy Sentence 2 & $0.67 \pm 0.39$ & $0.82 \pm 0.41$ & $7.51 \pm 1.42$ & $1.65 \pm 0.68$ & $95.99 \pm 55.98$ & $94.93 \pm 66.81$ \\
\hline Draw Circles & $1.55 \pm 1.17$ & $1.19 \pm 0.68$ & $5.57 \pm 1.09$ & $1.18 \pm 0.27$ & $107.91 \pm 42.72$ & $10.33 \pm 5.59$ \\
\hline Draw Circles & $1.27 \pm 0.74$ & $1.05 \pm 0.48$ & $5.53 \pm 1.59$ & $1.24 \pm 0.30$ & $128.82 \pm 34.12$ & $10.35 \pm 3.76$ \\
\hline
\end{tabular}

\section{Table 2.}

Intraclass correlation coefficient (ICC) (lower/upper bound of 95\% confidence interval [CI] in parentheses) of test-retest mean scores of handwriting kinematics and kinetics for six writing tasks completed with left hand by nine participants with permanent loss of function in previously dominant right hand.

\begin{tabular}{|c|c|c|c|c|c|c|}
\hline \multirow{2}{*}{ Task } & \multicolumn{2}{|c|}{ Mean Velocity } & \multicolumn{2}{|c|}{ Displacement } & \multirow{2}{*}{ On-Paper Time } & \multirow{2}{*}{ Force } \\
\hline & $x$ & $y$ & $x$ & $y$ & & \\
\hline Copy Alphabet & $\begin{array}{c}0.70 \\
(0.16 / 0.92)\end{array}$ & $\begin{array}{c}0 \\
(-0.61 / 0.63)\end{array}$ & $\begin{array}{c}0.79 \\
(0.35 / 0.95)\end{array}$ & $\begin{array}{c}0.06 \\
(-0.57 / 0.66)\end{array}$ & $\begin{array}{c}0.81 \\
(0.40 / 0.95)\end{array}$ & $\begin{array}{c}0.64 \\
(0.05 / 0.90)\end{array}$ \\
\hline Copy Date & $\begin{array}{c}0.81 \\
(0.41 / 0.95)\end{array}$ & $\begin{array}{c}0.38 \\
(-0.29 / 0.81)\end{array}$ & $\begin{array}{c}0.63 \\
(0.03 / 0.90)\end{array}$ & $\begin{array}{c}0.71 \\
(0.17 / 0.92)\end{array}$ & $\begin{array}{c}0.73 \\
(0.22 / 0.93)\end{array}$ & $\begin{array}{c}0.47 \\
(-0.19 / 0.85)\end{array}$ \\
\hline Copy Sentence 1 & $\begin{array}{c}0.67 \\
(0.11 / 0.91)\end{array}$ & $\begin{array}{c}0.19 \\
(-0.47 / 0.73)\end{array}$ & $\begin{array}{c}0.62 \\
(0.03 /-0.90)\end{array}$ & $\begin{array}{c}0.39 \\
(-0.29 / 0.82)\end{array}$ & $\begin{array}{c}0.88 \\
(0.60 / 0.98)\end{array}$ & $\begin{array}{c}0.43 \\
(-0.24 / 0.83)\end{array}$ \\
\hline Copy Sentence 2 & $\begin{array}{c}0.63 \\
(0.04 / 0.92)\end{array}$ & $\begin{array}{c}-0.01 \\
(-0.61 / 0.63)\end{array}$ & $\begin{array}{c}0.71 \\
(0.17 / 0.92)\end{array}$ & $\begin{array}{c}-0.33 \\
(-0.78 / 0.37)\end{array}$ & $\begin{array}{c}0.78 \\
(0.33 / 0.95)\end{array}$ & $\begin{array}{c}0.61 \\
(-0.02 / 0.89)\end{array}$ \\
\hline
\end{tabular}

Performance stability was noted by objective evaluators who visually discerned handwriting features (size, shape, slant, and style) and matched handwriting samples from test and retest sessions. The evaluators' ability to correctly match handwriting samples showed 100 percent success for three tasks: Copy Alphabet, Copy Sentence 1, and Copy Sentence 2. One evaluator correctly matched all nine pairs for the Compose a Sentence and Copy Date tasks, whereas the second evaluator correctly matched eight out of nine pairs for both tasks. Both evaluators matched eight out of nine pairs for the Draw Circles task. Figure 2 shows three participants' handwriting samples from the Copy Sentence tasks in the test and retest sessions.

If written-letters-per-minute is used as a marker of fluency of writing and fluency is extrapolated as a marker of writing competency, three participants performed 
(a)

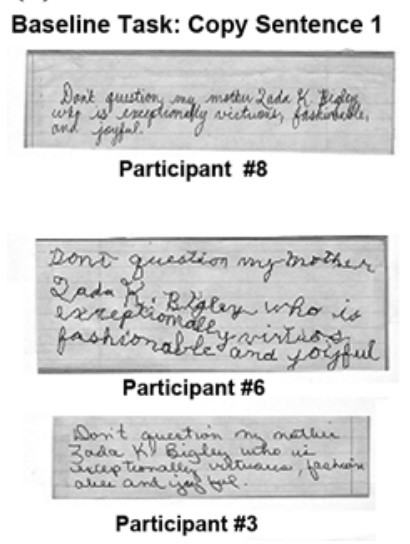

(b)
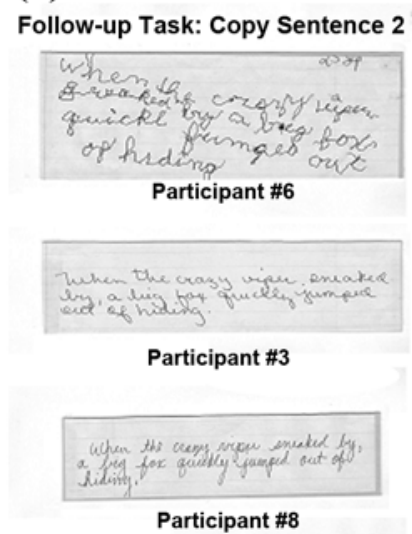

Figure 2.

Copy Sentence tasks at (a) baseline and (b) follow-up. Two objective evaluators examined all de-identified handwriting samples and were 100 percent accurate in correctly matching samples from all nine participants from both sentence-copying tasks. Differences in paper sizes reflect trimming to remove participant identifiers.

between a first- and third-grade fluency level, with a range of 17 to 48 letters per minute; the remaining six participants scored between an eighth- and ninth-grade level, with a range of 93 to 168 letters per minute. Table 3 shows the grade-level equivalent for writing performance for each participant. Table 3 also shows each participant's on-paper time for the Copy Alphabet task, with comparisons to reference normative values from the JTHF writing subtest. Three participants met writing performance standards according to normative data from the dominant hand, three participants met writing performance standards according to data from the nondominant hand, and three participants did not meet performance standards for either the dominant or nondominant hand.

\section{DISCUSSION}

Results of this pilot study captured writing performance stability within subjects, as noted by minimal differences between retest and test of group means for kinetic and kinematic parameters. The large SDs around group means reveal between-subject performance variability. The negative ICC values and the 95 percent confidence intervals (CIs) that include a zero value generally imply no correlation between test and retest sessions; however, given the minimal differences between test and

retest group means, the negative ICC values likely express large SDs captured statistically in the ICC values and CIs.

As task complexity increased, so did variability between test and retest sessions; for example, the Compose a Sentence task showed more variability between testing sessions than did the Draw Circles or Copy Date tasks. Likewise, performance of the three tasks that varied between sessions (Compose a Sentence, Copy Sentence 1, and Copy Sentence 2) was less consistent than performance on the tasks that remained the same (Draw Circles, Copy Date, Copy Alphabet). In this way, perhaps kinematic analysis is too sensitive a measure of performance on complex handwriting tasks and tasks that vary (even slightly) between testing sessions. However, measuring subjects' ability to copy and compose sentences and dates captures functional handwriting performance more than the meaningless copying of lines and circles.

The increased mean velocity in the $x$ and $y$ directions and greater force for all tasks at retest suggest more effort on task performance at retest. The longer on-paper times for Copy Alphabet, Copy Sentence 1, and Copy Sentence 2 imply the same conclusion: change in performance was caused by awareness of being tested, a testing effect referred to as the "Hawthorne effect" [23].

Visual analysis of handwriting samples is common among certified forensic document examiners, as well as occupational therapy practitioners administering traditional paper-and-pencil assessments in school settings. Visual analysis methods were applied in this pilot study as two independent evaluators matched test-to-retest handwriting samples on the basis of consistency in letter size, shape, slant, and overall style. So, while kinematic analysis was used to assess stability in the handwriting process, visual analysis assessed stability in the handwriting product.

Results of kinematic and visual analysis support the following conclusions: (1) despite instability of select kinematic and kinetic performance parameters, participants' written output was consistent (recognizably similar and therefore presumed stable) between test and retest sessions; (2) results of both analyses showed betweensubject variability; and (3) between-subject variability expressed itself in unique writing styles, which suggests an idiosyncratic nature of handwriting.

Adult-level writing demands mastery of fine motor coordination for basic writing fluency in order to liberate the brain to attend to higher order cognitive tasks [24]. 
Table 3.

Descriptive analysis of all participants.

\begin{tabular}{|c|c|c|c|c|c|}
\hline Sex & Age & $\begin{array}{c}\text { Time Since } \\
\text { Amputation (yr) }\end{array}$ & $\begin{array}{c}\text { Highest } \\
\text { Education Level }\end{array}$ & $\begin{array}{c}\text { Writing Performance } \\
\text { Grade-Level Equivalent }\end{array}$ & $\begin{array}{c}\text { On-Paper Time for Copy } \\
\text { Alphabet Task (s) }\end{array}$ \\
\hline $\mathrm{F}$ & 62 & 7 & High School & 8th & $28.54^{*}$ \\
\hline F & 59 & 16 & High School & 9th & 10.39 \\
\hline $\mathrm{F}$ & 65 & 46 & Associate Degree & $>9$ th & 14.06 \\
\hline F & 70 & 6 & Bachelor Degree & 9th & $37.08^{*}$ \\
\hline M & 58 & 7 & High School & $1 \mathrm{st}$ & $122.57^{\dagger}$ \\
\hline $\mathrm{F}$ & 29 & 4 & Master's Degree & $>9$ th & $23.77^{*}$ \\
\hline M & 61 & 40 & High School & $>9$ th & 22.53 \\
\hline M & 52 & 6 & 6th Grade & 2nd & $64.94^{\dagger}$ \\
\hline
\end{tabular}

Looking at grade-level equivalence for each participant's writing speed aroused concern for three participants who wrote at speeds comparable to first, second, and third graders, despite a significant amount of elapsed time since loss of dominant-hand function.

A recent adult survey found 92 million Americans with literacy levels at less than an eighth-grade level [25], and since the Flesch-Kincaid assessment rated the Copy Sentence 2 task at a 13.4 grade level, slow performance speed possibly reflected difficulty with adult-level literacy tasks rather than limits in the fine motor control needed for writing. Therefore, the on-paper time for the simple Copy Alphabet task was compared with adult reference normative values of the similar JTHF writing subtest. This comparison showed that the three participants with low grade-level writing speeds also did not meet performance standards for dominant or nondominant hand, confirming a motor, rather than cognitive, performance constraint.

A closer look at these participants substantiates the conclusion of a motor control, rather than literacy skill, constraint. All three participants reported at least a sixthgrade education and therefore were assumed to be capable of writing the alphabet in the Copy Alphabet task. One participant reported that he had written for less than 5 minutes per day since his amputation 3 years prior and another participant reported that he had not written since his amputation 7 years prior. The third participant reported that she had written each day since her amputation 6 years prior; although she had slow performance (second-grade equivalent), she wrote faster than the other two participants.

These findings align with other research that suggests handwriting is not an autoemergent skill but rather one that needs to be purposefully addressed [26-27]. For example, Eggers and Mennen discuss the phenomenon of handdominance transfer as a product of functional adaptation to accomplish ADL when motion and sensation are traumatically lost in the "main executor" arm and hand, and conjecture that skilled actions beyond those of an 8-yearold child require extensive, deliberate practice to facilitate transfer because of the necessary proficiency, speed, and agility [1]. In this study, all participants were independent in basic ADL; however, they had not all transferred handwriting skill at an adult proficiency level.

\section{Implications for Practice and Research}

Results showed that eight out of nine participants engaged in handwriting tasks daily, which supports the notion that handwriting remains a meaningful daily task and should be addressed in rehabilitation care plans. Results provide clinical value by establishing and describing a method for measuring functional handwriting skill. These methods may be replicated and extended to measure handwriting in other populations of interest. Providing adequate description of the experimental methods allows other therapists and researchers to pursue research studies in this avenue of inquiry. Further studies may generate improved measurement techniques and ultimately improved rehabilitation methods for therapists working with patients facing I-IHDT. 
Study results also inform clinicians about overall graphomotor performance consistency across tasks and kinematic parameters. While the sample is too small to establish normative data, the information can be used clinically; for example, a therapist working with a patient who has lost dominant-hand function may repeat the tasks and measure the variables that showed excellent reliability to monitor a change over time to evaluate therapeutic progress.

Results of this pilot study have guided two subsequent studies related to a 6-week transfer intervention that uses handwriting as the defining motor task of hand dominance. The results have influenced these intervention studies in three primary ways. First, a single-subject research design was chosen. This is a result of the large SDs around the group mean differences, the heterogeneity of the participants, and the difficulty obtaining a large sample size. Single-subject research avoids group analysis by using a rigorous experimental approach in which each participant is his or her own control. Second, much closer attention is being paid to personal factors that may influence performance, such as neuromusculoskeletal functions in the sole functioning limb (joint mobility, stability, power, tone, and endurance); cognitive functions of attention, memory, visual perception; and psychosocial factors such as insight into functional loss, past occupational experiences and future occupational goals, and motivation for transferring handwriting skill. And lastly, kinematic analysis proved valuable for simpler writing tasks, but traditional paperand-pencil metrics are being used to measure letters per minute and legibility in complex, adult-level handwriting tasks.

\section{Limitations}

Gaining access to a population of community-dwelling adults with permanent loss of dominant-hand function was difficult, resulting in a small and heterogeneous sample. A small sample prohibited statistical methods of regression analysis to discern variables, such as time since functional loss, that may contribute to the fine motor control necessary to establish stable movement patterns for handwriting. Because this was not a clinical study, access was unavailable to the participants' medical records and other health information that may have influenced motor performance. Similarly, clinical evaluations that may have been useful to this study, such as cognitive, sensory, motor, or strength assessments, were not performed. Finally, the concession to meet participants at convenient locations resulted in limited control over environmental constraints, such as time of day, lighting, noise/distractions, and room temperature. This may have contributed to between-subject variability.

\section{CONCLUSIONS}

When hand-injured patients face I-IHDT, they deserve evidence-based interventions to accelerate necessary handdominance transfer so they may be restored to full participation in ADL, work, and leisure pursuits. This study examined graphomotor performance as a marker of hand dominance in a distinct sample of adults who had lost dominant-hand function and discovered which kinematic and kinetic parameters were stable across time and across various functional writing tasks. This information has been useful in the design of ongoing clinical trials of an intervention to facilitate hand-dominance transfer. Research in this line of inquiry needs to be extended to advance initiatives in rehabilitation to minimize the severity of disability following dominant-hand injuries [28].

\section{ACKNOWLEDGMENTS}

\section{Author Contributions:}

Study concept and design: K. E. Yancosek, D. R. Mullineaux. Coordination of study subjects' meeting times: K. E. Yancosek. Acquisition of data: K. E. Yancosek.

Analysis and interpretation of data: K. E. Yancosek, D. R. Mullineaux.

Drafting of manuscript: K. E. Yancosek, D. R. Mullineaux. Critical revision of manuscript for important intellectual content: K. E. Yancosek, D. R. Mullineaux.

Statistical analysis: K. E. Yancosek, D. R. Mullineaux. Obtained funding: K. E. Yancosek.

Additional Contributions: Dr. Yancosek is now with the U.S. Institute of Environmental Medicine in Natick, Massachusetts.

Financial Disclosures: The authors have declared that no competing interests exist.

Funding/Support: This material was based on work supported by a \$5,000 Seed Grant from the University of Kentucky’s Center for Clinical and Translational Science.

Institutional Review: This pilot study was approved by the local institutional review board, and participants provided written informed consent.

Participant Follow-Up: The authors do not plan to inform participants of the publication of this study because contact information is unavailable. 


\section{REFERENCES}

1. Eggers IM, Mennen U. The EFFUL system (Evaluation of Function in the Flail Upper Limb) system. A ranking score system to measure improvement achieved by surgical reconstruction and rehabilitation. J Hand Surg Br. 1997; 22(3):388-94. [PMID: 9222925]

DOI:10.1016/S0266-7681(97)80410-X

2. Kimmerle M, Mainwaring L, Borenstein M. The functional repertoire of the hand and its application to assessment. Am J Occup Ther. 2003;57(5):489-98. [PMID: 14527110]

3. Walsh WW, Belding NN, Taylor E, Nunley JA. The effect of upper extremity trauma on handedness. Am J Occup Ther. 1993;47(9):787-95. [PMID: 8116769]

4. Frettlöh J, Hüppe M, Maier C. Severity and specificity of neglect-like symptoms in patients with complex regional pain syndrome (CRPS) compared to chronic limb pain of other origins. Pain. 2006;124(1-2):184-89. [PMID: 16730904]

5. Granville DA, Ehrman L, Perelle ID. Laterality survey: Questionnaire results. Mensa Bull. 1980;239:26.

6. Chu S. Occupational therapy for children with handwriting difficulties: A framework for evaluation and treatment. Br J Occup Ther. 1997;60:514-20.

7. Bonney MA. Understanding and assessing handwriting difficulty: Perspectives from the literature. Aust Occup Ther J. 1992;39(3):7-15. DOI:10.1111/j.1440-1630.1992.tb01751.x

8. Plakins Thornton T. Handwriting in America: A cultural history. New Haven (CT): Yale University Press; 1996.

9. Faddy K, McCluskey A, Lannin NA. Interrater reliability of a new handwriting assessment battery for adults. Am J Occup Ther. 2008;62(5):595-99. [PMID: 18826021]

10. Jebsen RH, Taylor N, Trieschmann RB, Trotter MJ, Howard LA. An objective and standardized test of hand function. Arch Phys Med Rehabil. 1969;50(6):311-19. [PMID: 5788487]

11. MacDermid JC, Tottenham V. Responsiveness of the disability of the arm, shoulder, and hand (DASH) and patientrated wrist/hand evaluation (PRWHE) in evaluating change after hand therapy. J Hand Ther. 2004;17(1):18-23.

[PMID: 14770134$]$

DOI:10.1197/j.jht.2003.10.003

12. Blades B, Mellis N, Munster AM. A burn specific health scale. J Trauma. 1982;22(10):872-75. [PMID: 7131607] DOI:10.1097/00005373-198210000-00012

13. Stratford PW, Binkley JM, Stratford DM. Development and initial validation of the upper extremity functional index. Physiother Can. 2001;52:259-67.

14. Andersen Hammond ER, Shay BL, Szturm T. Objective evaluation of fine motor manipulation-A new clinical tool. J Hand Ther. 2009;22(1):28-36. [PMID: 18950989] DOI:10.1197/j.jht.2008.06.006

15. Smeulders MJ, Kreulen M, Bos KE. Fine motor assessment in chronic wrist pain: The role of adapted motor control.
Clin Rehabil. 2001;15(2):133-41. [PMID: 11330758]

DOI:10.1191/026921501672958566

16. Chan SW, LaStayo P. Hand therapy management following mutilating hand injuries. Hand Clin. 2003;19(1):133-48.

[PMID: 12683451$]$

DOI:10.1016/S0749-0712(02)00140-3

17. Andree ME, Maitra KK. Intermanual transfer of a new writing occupation in young adults without disability. Occup Ther Int. 2002;9(1):41-56. [PMID: 12375007] DOI:10.1002/oti.155

18. Walker L, Henneberg M. Writing with the non-dominant hand: Cross-handedness trainability in adult individuals. Laterality. 2007;12(2):121-30. [PMID: 17365628] DOI:10.1080/13576500600989665

19. Klöppel S, Vongerichten A, Van Eimeren T, Frackowiak RS, Siebner HR. Can left-handedness be switched? Insights from an early switch of handwriting. J Neurosci. 2007; 27(29):7847-53. [PMID: 17634378] DOI:10.1523/JNEUROSCI.1299-07.2007

20. Lubrano V, Roux FE, Démonet JF. Writing-specific sites in frontal areas: A cortical stimulation study. J Neurosurg. 2004; 101(5):787-98. [PMID: 15540917]

DOI:10.3171/jns.2004.101.5.0787

21. Doak CC, Doak LG, Root JH. Teaching patients with low literacy skills. 2nd ed. Philadelphia (PA): J.B. Lippincott; 1996.

22. Graham S, Berninger VW, Weintraub N, Schafer W. Development of handwriting speed and legibility in grades 1-9. J Educ Res. 1998;92(1):42-52. DOI:10.1080/00220679809597574

23. Steele-Johnson D, Beauregard RS, Hoover PB, Schmidt AM. Goal orientation and task demand effects on motivation, affect, and performance. J Appl Psychol. 2000;85(5): 724-28. [PMID: 11055145]

DOI:10.1037/0021-9010.85.5.724

24. Connelly V, Dockrell JE, Barnett J. The slow handwriting of undergraduate students constrains overall performance in exam essays. Educ Psychol. 2005;25(1):99-107.

DOI:10.1080/0144341042000294912

25. Kutner M, Greenberg E, Baer J. A first look at the literacy of America's adults in the 21st century [Internet]. Washington (DC): National Center for Education Statistics; 2005 [updated 2005; cited 2010 Jan 15]. Available from: http:// nces.ed.gov/NAAL/PDF/2006470.PDF.

26. Jones D, Christensen CA. Relationship between automaticity in handwriting and students' ability to generate written text. J Educ Psychol. 1999;91:44-49.

DOI:10.1037/0022-0663.91.1.44

27. Graham S. Issues in handwriting instruction. Focus on exceptional children. 1992;25(2):1-14.

28. Trybus M, Lorkowski J, Brongel L, Hladki W. Causes and consequences of hand injuries. Am J Surg. 2006;192(1): 52-57. [PMID: 16769275] DOI:10.1016/j.amjsurg.2005.10.055 
Submitted for publication April 28, 2010. Accepted in revised form September 13, 2010.

This article and any supplementary material should be cited as follows:

Yancosek KE, Mullineaux DR. Stability of handwriting performance following injury-induced hand-dominance transfer in adults: A pilot study. J Rehabil Res Dev. 2011;48(1):59-68.

DOI:10.1682/JRRD.2010.04.0074

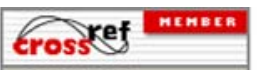

CROSSREF ORG 